\title{
Un nuevo modelo territorial de las zonas de protección natural periurbanas en la microcuenca del río Tarqui
}

\author{
A new territorial model of peri-urban areas of natural \\ protection in the micro-watershed of the Tarqui River
}

Por:

Diana Alejandra

Orellana Valdez

Universidad de Cuenca

Recibido:

15 de febrero 2012

Aceptado:

15 de Marzo 2012

\section{Resumen:}

La ordenanza que sanciona el Plan de Ordenamiento Territorial del cantón Cuenca, vigente desde el año 2003, determina el suelo no urbanizable en el área de influencia inmediata a la ciudad debido a algunos factores, uno de ellos es el valor natural que poseen cinco zonas bien delimitadas denominadas Zonas de Protección Natural (ZPN), en éstas zonas se restringe toda actividad que implique la artificialización del suelo, prohibiéndose también su fraccionamiento; sin embargo, hasta ahora se muestran vulnerables por el crecimiento urbano al no existir un plan alguno que defina su gestión.

Bajo esa circunstancia, se aplicó la metodología planteada por el doctor Domingo Gómez Orea para la elaboración del Plan de Ordenamiento Territorial de tres de las cinco ZPN periurbanas: Loma de Huizhil, Cerro Monjas, Agua Santa y el área de influencia inmediata -la microcuenca del río Tarqui- común a los tres territorios, la misma que se caracteriza por armonizar las actividades y aspiraciones humanas con la capacidad de acogida de cada territorio.

Palabras clave: Modelo territorial, espacios de protección natural, Cuenca, Ecuador.

\begin{abstract}
:
The Ordinance that punishes the Territorial ordering Plan of canton Cuenca, existing since 2003, determines the non-developable land in the area of influence close to the city because some factors, one of them is the natural value possessing five well-defined areas called Areas of Natural Protection (ZPN), in these areas is restricted all activity involving the artificialization of land, banning also their fractionation. However, so far are vulnerable by urban growth because there is no any plan that defines their management.

Under that circumstance, we applied the methodology proposed by Dr. Domingo Gómez Orea to the elaboration of the Territorial ordering Plan used in three of the five ZPN peri-urban areas: Loma de Huizhil, Cerro Monjas, Agua Santa and the area of close influence - the micro-watershed of the Tarqui river-common to the three territories, the same that is characterized by harmonize activities and human aspirations with the reception capacity of each territory.
\end{abstract}

Keywords: Territorial model, natural protection areas, Cuenca, Ecuador. 


\section{Introducción:}

\subsection{Hacia una ciudad sustentable}

"Las ciudades son los sistemas que mayor impacto generan al Planeta y por esto, sabemos que la batalla de la sostenibilidad la vamos a ganar o perder en base a la organización y la gestión urbanas que desarrollemos a partir de ahora" (Ministerio del Ambiente Español, 2007, Tomo I: 12) Tal afirmación es indiscutible, pues es un hecho bien conocido que el ritmo de explotación de los recursos naturales y la producción de desechos expulsados al ambiente superan la capacidad de regeneración de la naturaleza; sin embargo, la posibilidad de recuperar el equilibrio es real, los avances científicos y técnicos así lo demuestran, y las dificultades y limitaciones son más bien de orden político y social.

Para gestionar una ciudad sustentable es preciso concebirla en un escenario futuro óptimo, como un ecosistema natural; ahora bien, si la ciudad no se autorregula como la naturaleza, se deberá planificar y gestionar buscando disminuir la brecha entre lo que producimos y lo que se consume del medio físico mediante el uso eficiente de los recursos. Un entendimiento ecosistémico de la ciudad precisa mirar con visión metropolitana incluyendo al suelo urbano, periurbano y rural con sus interrelaciones de dependencia; es decir, "una ciudad sólo puede ser entendida como un ecosistema completo si se consideran incluidos en él los ambientes de entrada y salida" (Salvador, 2003: 136 ) Con esa premisa, el suelo periurbano y el suelo rural recuperan su valor hasta ahora perdido por calificarlo como "urbanizable" o suelo útil y "no urbanizable".

\subsection{El fenómeno periurbano genera una ciu- dad dispersa}

El fenómeno periurbano es la metamorfosis que experimentan las zonas rurales inmediatas en nuevas superficies de expansión de las ciudades, con usos propiamente urbanos que se contraponen a los rurales primarios. (Ferras, 2000) Expansión que es consecuencia del modelo de desarrollo capitalista actual y que genera un crecimiento disperso y desordenado que consume el suelo de una manera insensible con el medio físico, situación que pone en riesgo a los territorios de valor natural -así determinados por su aptitud agrícola, forestal o por albergar especies endémicas. Ese crecimiento urbano actual no responde más que a los intereses económicos particulares de los propietarios del suelo o de ciertos sectores del mercado, como el inmobiliario.

El modelo de "ciudad dispersa" es un resultado del fenómeno periurbano y es insostenible. Así por ejemplo, el aumentar la distancia de los recorridos diarios implica el uso intensivo del vehículo privado en detrimento de la calidad del aire y los conflictos de tráfico; el abastecimiento de servicios básicos de la nueva población en la periferia exige contar con más recursos, ya sean éstos naturales, económicos y/o materiales. En una ciudad dispersa los usos se homogeneizan y la ciudad pierde complejidad, se construyen perímetros de urbanizaciones residenciales aisladas y en la periferia, que rápidamente demandan equipamientos y usos complementarios de intercambio y de servicios para la vivienda; y finalmente, se genera la sectorización y la segregación social al determinar ciertos sitios como exclusivos para aquellas personas con mayor capital económico y viceversa. (Rueda, 2003)

Al fenómeno periurbano se lo denomina también: suburbanización, contraurbanización o rururbanismo; sin embargo, esos conceptos no están completamente establecidos porque tratan de definir un fenómeno complejo que está en constante evolución, lo que indica además que el problema conceptual no está resuelto aún.

En la ciudad de Cuenca, el crecimiento disperso tiene además de las consecuencias negativas antes expuestas, otras más particulares íntimamente relacionadas con su topografía. Al estar ubicada en un valle interandino rodeado por la cordillera Occidental, Oriental y por nudos montañosos al Norte y al Sur, el suelo periurbano y rural es un conjunto de montañas menores que envuelve la ciudad, situación que condiciona la expansión urbana. Las consecuencias particulares del crecimiento disperso en Cuenca se caracterizan por la incompatibilidad de las actividades con su medio físico, así por ejemplo: la baja calidad estética de las edificaciones, la imposibilidad de abastecer las necesidades básicas, la disminución de la capa vegetal y de especies endémicas y nativas, la erosión hídrica del suelo, el aumento de riesgo de inestabilidad geológi$\mathrm{ca}$, las pérdidas materiales y humanas por deslizamientos en época de invierno, el deterioro del paisaje y la pérdida del suelo con aptitudes agrícolas, etc., son problemas que disminuyen la calidad de vida de los habitantes, realidad que a mediano y largo plazo transformará a Cuenca en un sistema territorial aún más complejo e insostenible por su crecimiento descontrolado y su difícil gestión.

Más allá del qué se implante o qué actividades se ejecuten sobre el suelo periurbano cuencano, es el cómo esas actividades se desarrollan en un territorio, el que exige un mayor esfuerzo por parte de los propietarios y proyectis- 
tas, siempre con una misma perspectiva, un enfoque ecosistémico de ciudad que ejecute toda acción conciliando las necesidades humanas con las aptitudes del medio natural, de manera tal que no atente contra la seguridad de los actuales y futuros habitantes. En contraposición a ese concepto ideal, la especulación del suelo urbano en Cuenca es real y es la principal causante del fenómeno periurbano, los altos costos que tiene el suelo limitan su compra y obligan a las nuevas familias a vivir en zonas cada vez más alejadas del área urbana, lo mismo sucede con las inmobiliarias y sus urbanizaciones, que en busca de predios más grandes y de mejores ingresos, ofrecen viviendas en suelo periurbano, configurando así una ciudad dispersa.

\subsection{Las zonas de protección natural periurbanas: cinturones o anillos verdes}

Teóricamente a las zonas de valor natural periurbanas se las denomina cinturones o anillos verdes, éstos pueden ser hortícolas o bosques, el concepto será el mismo: formar un sistema de vegetación alrededor de una ciudad, que consistirá en identificar, estudiar y conservar determinados puntos o islas con recursos ubicados en la periferia de valor natural unidos mediante corredores verdes, cuyo indispensable, pues son los encargados de enlazar no sólo físicamente los puntos de valor ecológico, sino que permiten además el desplazamiento de organismos entre las islas de forma lineal y conectora, un ejemplo claro de corredor verde son las cuencas de inundación de los ríos.

Entre las funciones que cumplen los anillos verdes se conoce que: "expanden el área de hábitats aislados; conectan a poblaciones de especies de flora y fauna (intercambio genético y efecto de rescate) y así aumentan el tiempo de residencia de poblaciones de plantas y animales en un mismo sitio; permiten el movimiento diario, estacional o migratorio de los animales; son filtros o barreras al movimiento de ciertas especies y fuentes y piletas para ciertos efectos bióticos y ambientales; regulan el clima local y actúan como barrera protectora ante eventos climáticos; promueven las interacciones de plantas y animales (dispersión de semillas, polarizadores, etc.) y ayudan a los procesos ecológicos que tienen como vectores de dispersión a los animales, el viento o las corrientes de agua; crean un gradiente entre las unidades de máxima protección -áreas protegidas-y las áreas de máxima explotación, aprovechamiento y asentamientos; son una herramienta exitosa en el manejo y protección de las cuencas hidrográficas, ya que regulan los flujos hidrológicos, previenen inundaciones y proveen agua limpia; logran que las áreas protegidas dentro del corredor se administren bajo la misma concepción de desarrollo sostenible; mejoran la planificación y gestión de recursos naturales y promueven actividades económicas relacionadas a la conservación; promueven investigaciones biológicas y socioeconómicas que pueden revelar nuevas herramientas para reducir las amenazas a la biodiversidad; promueven la cohe- sión y comunicación entre culturas; ayudan a ganar un peso político considerable que contribuya a que los intereses de personas e instituciones vinculados al Corredor puedan llegar a convertirse en políticas de Estado, en la medida que apunten a la conservación de la biodiversidad y al uso sustentable de los recursos" . (USAID, 2009: 6)

Finalmente, mitigan el cambio climático porque retienen dióxido de carbono, reducen la contaminación acústica y lumínica, protegen el suelo de la erosión, purifican el aire, el suelo y el agua, ofrecen productos agrícolas y silvícolas y constituyen un óptimo espacio para el deporte y el ocio; en definitiva mejoran la calidad de vida. (Nilson.,1997)

\subsection{Marco legal de las zonas de protección natural periurbanas en el Ecuador}

En el Ecuador no existe una legislación que reconozca el valor de las zonas naturales periurbanas de manera específica; sin embargo, en la Constitución de 2008 se establece que la planificación territorial del área rural es una competencia municipal, así como el preservar, mantener y difundir el patrimonio natural del cantón, además como una estrategia para alcanzar el Buen Vivir, la Constitución establece que: "Se declara de interés público la preservación del ambiente, la conservación de los ecosistemas, la biodiversidad y la integridad del patrimonio genético del país, la prevención del daño ambiental y la recuperación de los espacios naturales degradados" (Constitución del Ecuador, 2008) Según el Código Orgánico de Organización Territorial, Autonomía y Descentralización (COOTAD), es responsabilidad de las municipalidades: "Establecer el régimen de uso del suelo urbanístico, para lo cual determinará las condiciones de urbanización, parcelación, lotización, división o cualquier otra forma de fraccionamiento de conformidad con la planificación cantonal, asegurando porcentajes para zonas verdes y áreas comunales" (Asamblea Constituyente del Ecuador, 2011)

Esas son algunas directrices generales referentes al ámbito de planificación que llevan a afirmar que la Municipalidad de Cuenca es la responsable de planificar las ZPN periurbanas motivo de estudio. La presente investigación elabora un plan de ordenamiento territorial (POT) que propone un modelo de gestión piloto para proteger y conservar estas zonas valiosas, de manera que al mismo tiempo ellas se transformen en hitos de modelos sustentables en la ciudad en íntima relación con el área urbana. 


\begin{tabular}{l} 
ETAPA I: ANTECEDENTES TEÓRICOS \\
ETAPA III: LEVANTAMIENTO DE PROBLEMAS \\
\hline ETAPA III: DELIMITACIÓN DELÁREA DE ESTUDIO \\
\hline ETAPA IV: DIAGNÓSTICO
\end{tabular}

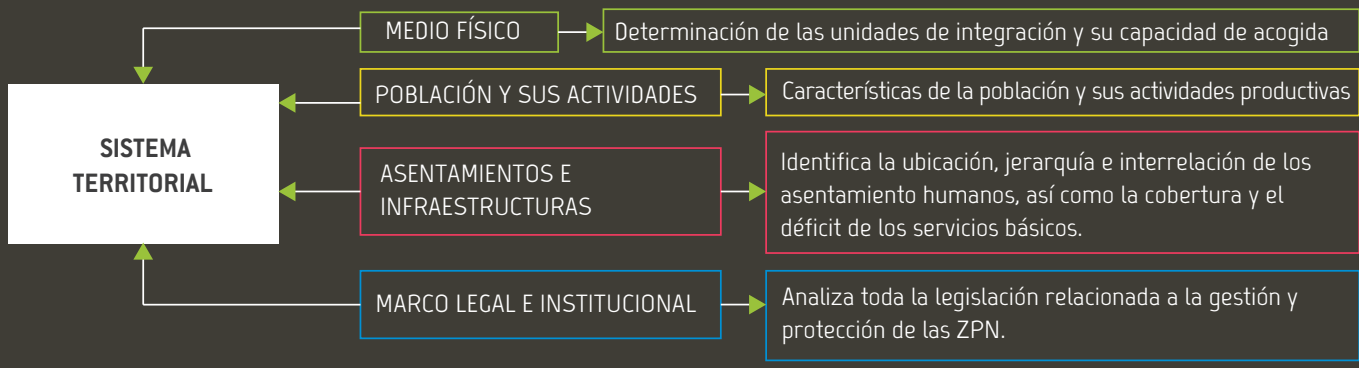

\begin{tabular}{|c|c|}
\hline ETAPA III: DELIMITACIÓN DEL ÁREA DE ESTUDIO & $\begin{array}{l}\text { Determinación del modelo territorial al identificar problemas, amenazas, } \\
\text { fortale zas, debilidades y oportunidades. Se trazan los posibles } \\
\text { escenarios futuros. }\end{array}$ \\
\hline ETAPAIV: DIAGNÓSTICO & $\begin{array}{l}\text { Propuesta de un nuevo modelo territorial para cuya ejecución se } \\
\text { determina una normativa reguladora de uso y ocupación de suelo, un } \\
\text { modelo de gestión, los programas y proyectos. }\end{array}$ \\
\hline
\end{tabular}

Gráfico № 1. Etapas de la investigación

\section{Método:}

El estudio se desarrolla en seis etapas y bajo la metodología planteada por el doctor Domingo Gómez Orea, cuyos principios se han seguido a través de su libro titulado: "Ordenamiento Territorial" (Ver Gráfico No. 1).

En él, se parte por entender al territorio como un sistema compuesto por cuatro subsistemas íntimamente relacionados entre sí: el medio físico, la población y sus actividades, el sistema de asentamientos e infraestructuras y el marco legal e institucional. En primera instancia se elabora un diagnóstico sectorial que estudia cada uno de los subsistemas territoriales, posteriormente se sintetizan los resultados obtenidos individualmente de tal forma que se crea el modelo territorial actual sobre el que se identifican problemas, fortalezas, debilidades, oportunidades, amenazas y un pronóstico de posibles escenarios futuros.

En segunda instancia se elabora el plan de ordenamiento propiamente dicho, que se compone de un sistema de objetivos que pretende revocar los as- pectos negativos y fortalecer los positivos del modelo territorial actual para definir un nuevo modelo territorial que se transformará en el objetivo a cumplir a mediano y largo plazo. Para la consecución del modelo territorial objetivo se elabora una normativa reguladora de uso y ocupación del suelo, programas con sus respectivos proyectos y un modelo de gestión.

El punto neurálgico de la metodología planteada reside en los resultados del diagnóstico sectorial del medio físico, en el cual se determinan las unidades territoriales de integración y su capacidad de acogida en base a las condiciones físicas del suelo, que con pocos cambios posteriormente en la fase del plan, se transforman en las categorías de ordenación que definen la asignación del uso y sus características de ocupación; es decir, las actividades propuestas en el área de estudio concilian las aptitudes del territorio con las necesidades de la población para lograr los objetivos que idealiza el plan: alcanzar un modelo de vida más sustentable.

Debido a la realidad multidisciplinaria que identifica a un plan de ordenamiento territorial, durante el proceso se acude a la asesoría de algunos técnicos especialistas. 


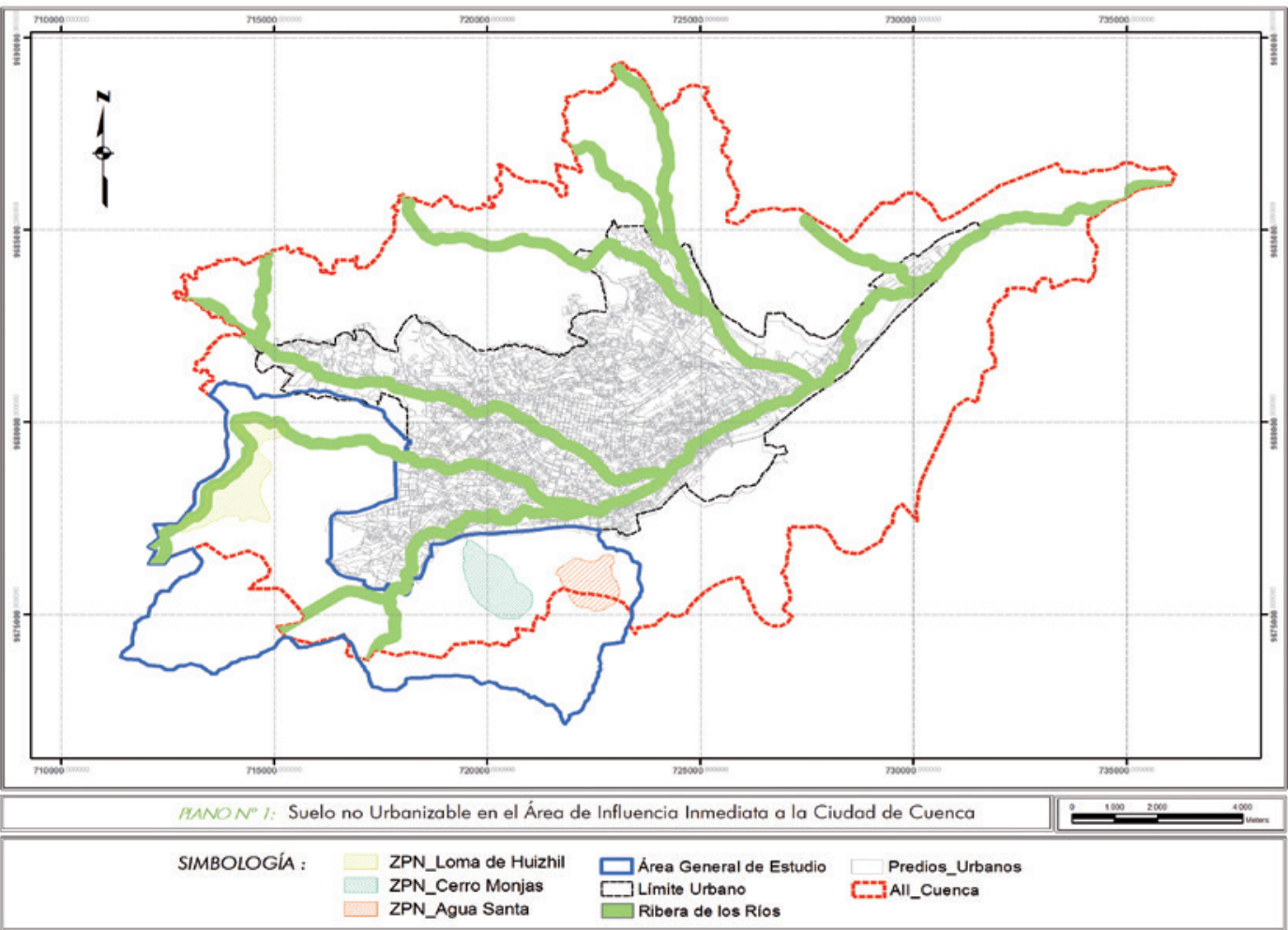

Plano $\mathbf{N}^{\circ} 1$.

Zonas de protección natural y su área de influencia inmediata

\section{Conclusiones:}

\subsection{Resultados del diagnóstico}

A continuación se describen cronológicamente las etapas más importantes y decisivas de la investigación que llevaron a trazar la propuesta de un nuevo modelo territorial.

\subsubsection{Delimitación del área de estudio}

Las ZPN del área de influencia inmediata de la ciudad han sido definidas como tales en la ordenanza que sanciona el Plan de Ordenamiento Territorial del cantón Cuenca, vigente desde el año 2003; su establecimiento y delimitación radican en los estudios realizados por el Proyecto PRECUPA (Proyecto de prevención de desastres naturales en la cuenca del río Paute) ejecutado en mayo de 1999. De las cinco zonas denominadas como áreas de protección natural. para el estudio se escogen tres, las más considerables en extensión: la loma de Huizhil con 256 ha, el cerro Monjas con 197 ha, Agua Santa con 149 ha, y su área de influencia inmediata la microcuenca del río Tarqui con 4586 ha. La decisión de establecer a la microcuenca como área de influencia inmediata se fundamenta en la necesidad de entender a las ZPN de una manera integrada al medio y no de forma aislada. A ello se suma el interés de encontrar la mayor cantidad de relaciones entre las tres ZPN para en una etapa posterior realizar propuestas con una visión sistémica que contemple las relaciones entre todos los componentes del territorio. (Ver Plano $N^{\circ} 1$, Fotografías $\left.N^{\circ} 1,2,3\right)$.

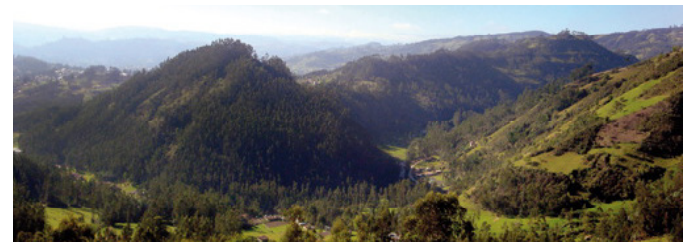

Fotografía $N^{\circ}$ 1. Zona de protección natural: Loma de Huizhil. Fuente: Autora de tesis

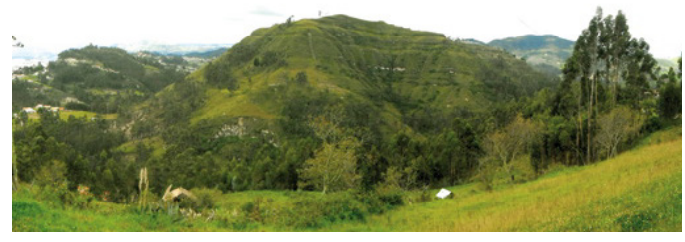

Fotografía $\mathbf{N}^{\circ} 2$. Zona de protección natural: Cerro Monjas. Fuente: Autora de tesis

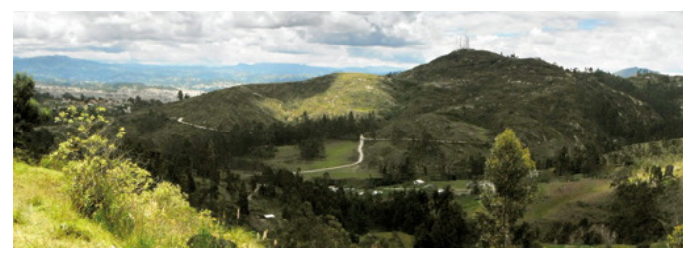

Fotografía $\mathbf{N}^{\circ}$ 3. Zona de protección natural: Agua Santa. Fuente: Autora de tesis 


\subsubsection{Determinación de las unidades de integración y su capacidad de acogida}

En el diagnóstico se analiza el territorio identificando todas las características físicas; para la determinación de las unidades de integración se consideran aquellas más importantes y que representan las aptitudes del territorio tales como: el suelo agrícola, suelo con valor paisajístico, arqueológico, las zonas de vegetación endémica, el suelo con pendiente superior al 30\% y finalmente los usos del suelo.

Según el último registro (año 2001), todos los mapas creados en cada caso se sobreponen entre sí de tal manera que resultan cuarenta unidades de integración. Una vez determinadas y nombradas las unidades se procede a caracterizarlas y valorarlas; según la metodología del doctor Gómez Orea, son cuatro componentes los que deben ser considerados para ello: valor ecológico, valor productivo, valor paisajístico y valor científico/ cultural, analizados de manera profunda en la etapa de diagnóstico. Para cada uno de ellos se considera cinco puntos, dando un total máximo de veinte puntos; el objetivo es identificar a las unidades de mayor valor para su protección. Los resultados de la valoración demuestran que las ZPN, entre otros territorios, tienen los puntajes más altos.

Luego se procede a determinar la capacidad de acogida, que consiste en asignar las actividades a ordenar con la mayor pertinencia posible a cada unidad de integración, considerando el menor impacto negativo y la máxima coincidencia de aptitud del territorio para su aprovechamiento sustentable. Para encontrar esa armonía o incompatibilidad se elabora una matriz de doble entrada: las unidades de integración con su valoración desplegada de manera vertical y las actividades a ordenar de manera horizontal; en las celdas de cruce se indica el nivel de impacto o de aptitud de cada actividad sobre el territorio.

\subsubsection{Síntesis de diagnóstico: Proble- mática del modelo territorial ac- tual de las ZPN y su área de in- fluencia inmediata}

Luego del análisis de diagnóstico se realiza un levantamiento de problemas y se hace uso de un grafo de relación causa-efecto; así se conclu- ye que los problemas primigenios y por ende de mayor importancia son los de orden legal, administrativo y de gestión; resulta ser sin duda, que la debilidad de la planificación territorial como disciplina es la etapa de gestión de un POT, pues por su propia condición socio-territorial y de orden público, se ve involucrada irremediablemente con intereses políticos. Antes de la Constitución de 2008 no estaba clara la competencia de las municipalidades sobre el suelo rural, eso ha desencadenado una urbanización de la periferia sin control alguno, situación que lleva a suponer que después de la legislación de 2008 se ha tomado una actitud responsable de asumir dicha competencia; sin embargo, ese no es el caso, aún ahora el conocimiento sobre las graves consecuencias de la periurbanización y su tratamiento como un problema clave a tratar en pos de un desarrollo urbano sustentable se ven lejanos. Esa realidad desarrolla toda una red de problemas sobre el territorio, de la cual nos interesa destacar la tendencia a la urbanización de las ZPN periurbanas (Ver Gráfico $N^{\circ} 2$ ). 


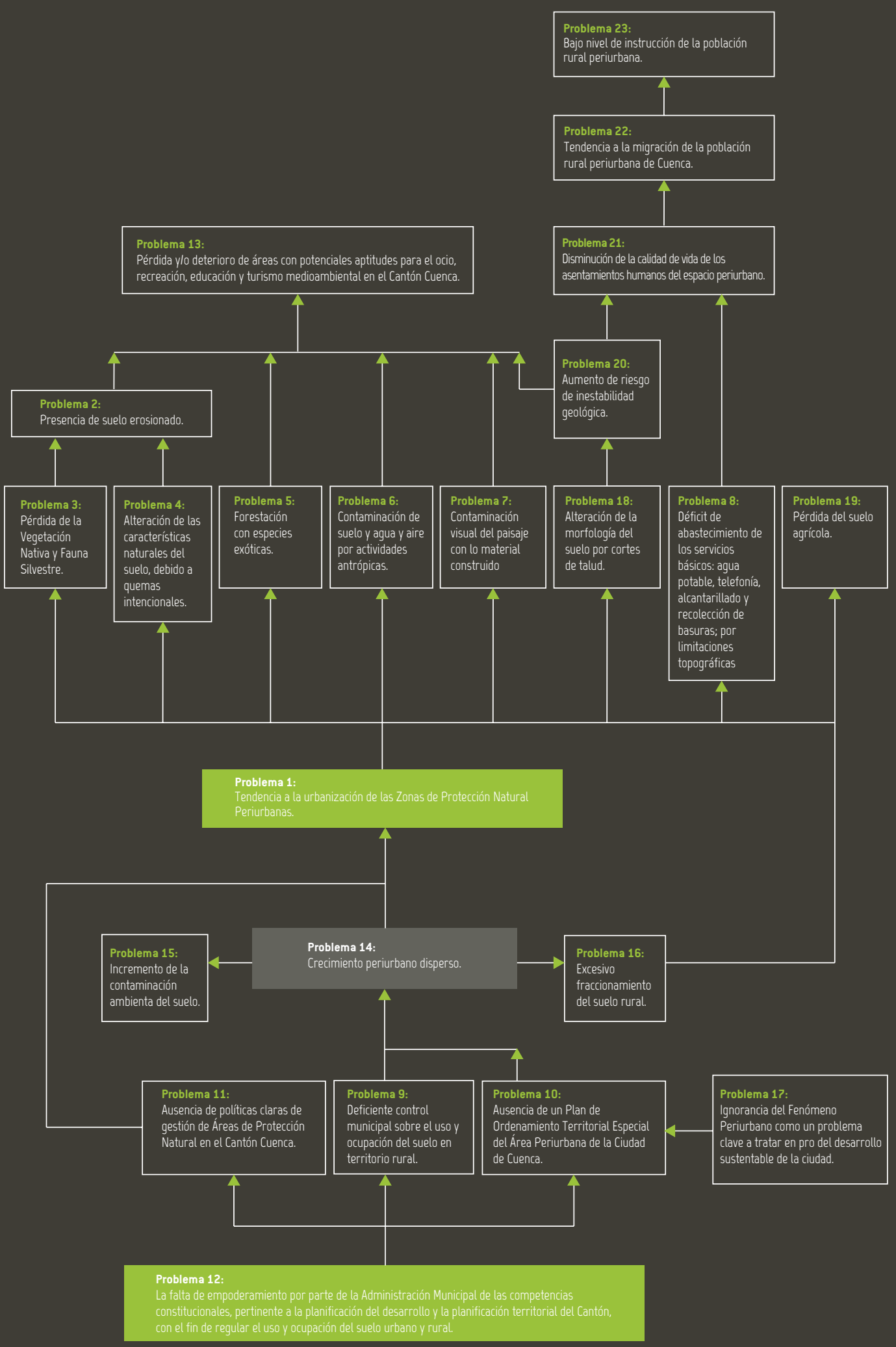

Gráfico $\mathbf{N}^{\circ}$ 2. Problemas del actual sistema territorial: Grafo de relación causa-efecto 


\subsection{Plan de Ordenamiento Territorial de la ZPN Periurbanas en la micro- cuenca del río Tarqui}

\subsubsection{Modelo territorial objetivo}

Una vez identificado el funcionamiento del sistema territorial actual del área de estudio, se procede a trazar el modelo territorial objetivo; según el doctor Gómez Orea, los elementos que configuran el modelo territorial objetivo son: a) submodelo de ordenación del medio físico correspondiente a las categorías de ordenación y b) submodelo de población, poblamiento e infraestructuras que comprende la distribución de los núcleos de población en el espacio, la jerarquía de los núcleos de población, los canales de relación, las conexiones con la zona externa y la localización de actividades secundarias y terciarias. Todo ello se resume en el Plano $\mathrm{N}^{\circ} 2$.

El modelo de ordenación del medio físico se resume en trece categorías de ordenación, las cuales son el resultado del análisis de la capacidad de acogida de las cuarenta unidades de integración, y son las siguientes:

A. Zona de conservación estricta: Comprende el $10,7 \%$ del territorio y corresponde, según el sistema determinado por la Unión Internacional para la Conservación de la Naturaleza (UICN), a la "Categoría lb: Área protegida manejada principalmente con fines científicos o con fines de protección de la naturaleza" (UICN, 1984 Sus objetivos son: asegurar que las generaciones futuras tengan la oportunidad de disfrutar y comprender el valor de zonas que han permanecido en gran medida inalteradas por la actividad humana durante un prolongado período de tiempo; mantener atributos y calidades naturales esenciales del medio ambiente a largo plazo; permitir el acceso del público de manera tal que contribuya con el bienestar físico y espiritual de los visitantes y preserven los atributos naturales de la zona para las generaciones actuales y futuras. (UICN, 1984) (Ver Plano $N^{\circ} 2$ ).

B. Zona de conservación activa con fines forestales: Correspondiente al $8,5 \%$ de la superficie total del área general de estudio, esta categoría se asigna a aquellos suelos con fuertes pendientes cuyas aptitudes son las actividades forestales. Las funciones que ésta plantea cumplir son: invertir el proceso de deforestación nativa, contribuir a la formación de ozono al aire de la ciudad, satisfacer la demanda local de productos forestales maderables y no maderables, fomentar la investigación científica en los campos de la biología e ingeniería genética, implementar el sistema de "Servicios Ambientales" como parte del modelo de gestión de las ZPN. Si bien en el área general de estudio, el porcentaje de suelo correspondiente a esta categoría no es muy representativo, al ampliar el análisis a todo el espacio periurbano de la ciudad, aumentarán las zonas con tales características y contribuirá a consolidar una ciudad sustentable (Ver Plano $\mathrm{N}^{\circ} 2$ ).

\section{Zonas de conservación activa con fines educativos- ambien-} tales: Comprende el $4 \%$ del territorio con una superficie de 207,86 ha. La educación ambiental consiste en crear conciencia de los problemas medioambientales locales y mundiales, así como generar las soluciones pertinentes a esos problemas. Por lo tanto, esta categoría recogerá las actividades afines con esos objetivos y las ubicará sobre su territorio. La educación ambiental contempla dos lineamientos generales, el primero consiste en entender los componentes de la naturaleza y sus interacciones (ecosistema), el segundo estudia cómo influyen las actividades antrópicas sobre esos procesos naturales; de esos lineamientos se puede posteriormente plantear soluciones para evitar fenómenos negativos consecuentes de las actividades humanas como la contaminación, erosión, pérdida del suelo agrícola, etc., que ya son problemas puntuales. (Internet, 2012) (Ver Plano N²).

D. Zona de conservación activa con fines histórico- culturales: Comprende el 9,4\% del territorio que corresponde a una superficie de 485,81 ha e incluye las ZPN: Cerro Monjas y Agua Santa. La conservación activa en este caso consiste en la investigación histórica de los vestigios arqueológicos aqu encontrados, restaurando y recreando los espacios arquitectónicos que revelan la forma de vida de los asentamientos cañaris desarrollados entre los años 500 d. C. a 1480 d. C. tales espacios no sólo se refieren a los restos de edificaciones encontrados, sino como en el caso del Cerro Monjas, en donde su morfología ha sido alterada en forma de terrazas por la mano humana, se podría descifrar de manera importante la forma de concebir la antigua ciudad de Guapondelig y de entender la cosmovisión de las culturas nativas preincas, así como la importancia que estos hitos o montañas tenían en esa época (Ver Plano $\mathrm{N}^{\circ} 2$ ).

Por otro lado, el cerro Monjas constituye además un hito de importancia natural por la presencia de vegetación arbustiva nativa y también de valor paisajístico por su ubicación a 310 $\mathrm{m}$ sobre la ciudad con una cuenca visual de $360^{\circ}$. En este sentido, retomando la categorización de las ZPN planteada por la UICN, la zona de conservación activa con fines históricoculturales se inscribe en la Categoría III: Monumento Natural: área protegida manejada principalmente para la conser- 
Un nuevo modelo territorial de las zonas de protección natural periurbanas...

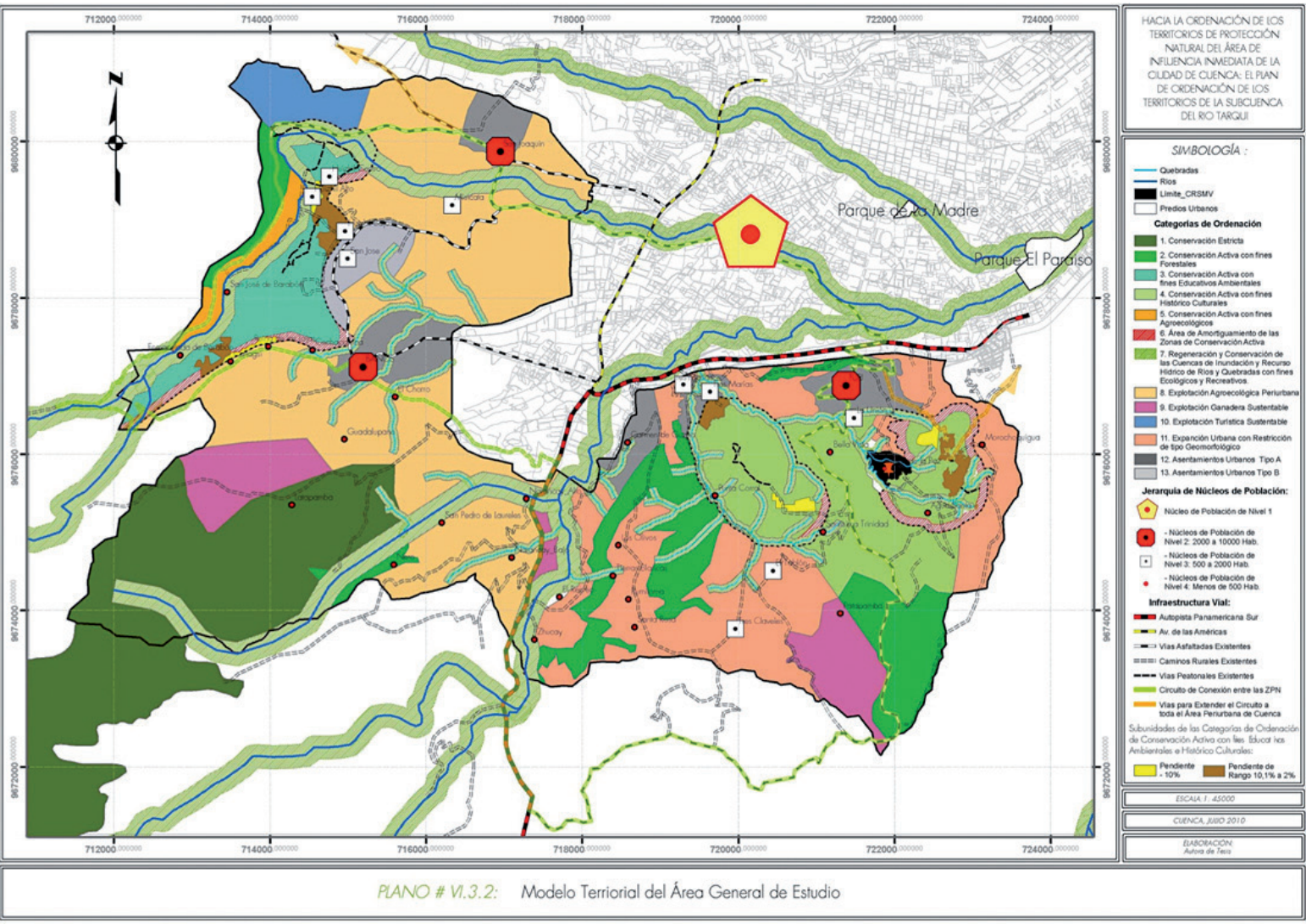

Plano $\mathbf{N}^{\circ}$ 2. Nuevo modelo territorial del área general de estudio

vación de características naturales específicas. (UICN, 1984) (Ver Plano $\mathrm{N}^{\circ} 2$ ).

\section{E. Zona de conservación activa con fines agroecológi-} cos: Corresponde al 1,1\% del territorio con una superficie de 56,27 ha. La agroecología es la aplicación de los conceptos y principios de la ecología al diseño, desarrollo y gestión de sistemas agrícolas sostenibles (Acosta. Rodríguez, 2005) Este nuevo enfoque científico introduce tres elementos que resultan claves: la preocupación medioambiental, una perspectiva ecológica y la preocupación social.

El objetivo de esta categoría es la enseñanza de la agroecología mediante su práctica en el territorio, dirigida a los agricultores propietarios de los predios. El aporte con el conocimiento tecnológico que puedan prestar las facultades de Agronomía de las universidades de la ciudad será de gran importancia para su desarrollo. De manera paralela y a menor escala se asignarían zonas de prácticas de producción agroecológica para alumnos de primaria y secundaria, cuya finalidad será impulsar el desarrollo rural y que los jóvenes se vinculen, a largo plazo, en las buenas prácticas agrícolas y así evitar su pérdida a futuro.
El territorio que conforma esta categoría es parte de la cuenca de inundación del río Yanuncay, es decir $150 \mathrm{~m}$ desde su eje. Dado que las actividades agrícolas ecológicas no alteran de manera radical el suelo, -esta zona tiene alto valor productivo y en la actualidad existen cultivos-, se permite dicha actividad considerándola como compatible (Ver Plano $\mathrm{N}^{\circ}$ 2).

F. Áreas de amortiguamiento de las zonas de conservación activa: Corresponden al $2 \%$ del territorio que comprenden 106,04 ha. Son los territorios adyacentes de las áreas naturales o zonas cuyas categorías son las de Conservación Activa. Su función es proporcionar una protección adicional que garantice el cumplimiento de los objetivos de conservación y mitigue las amenazas causadas por las actividades admisibles de los territorios vecinos y que puedan ser un riesgo para el suelo conservado. Por tales razones se prohíbe en general la edificación de cualquier tipo de construcciones en estos territorios, a excepción de aquellos relacionados con los usos permitidos en las categorías de Conservación Activa, como centros de interpretación, museos, etc. Se permiten también actividades primarias relacionadas con la producción agrícola y el pastoreo (Ver Plano $\mathrm{N}^{\circ} 2$ ). 
G. Zonas de regeneración y conservación de las cuencas de inundación y del recurso hídrico de ríos y quebradas con fines ecológicos y recreativos: Representa el 15,6\% que corresponde a una superficie de 807,72 ha del área general de estudio. Es muy frecuente encontrar edificaciones en estas zonas, el mayor inconveniente es el riesgo de inundaciones que amenaza la seguridad de sus habitantes y las pérdidas materiales que produce la crecida del caudal de ríos y quebradas. EI recurso hídrico se contamina conforme pasa por los territorios con presencia de asentamientos humanos, y con ellos la evacuación directa de aguas servidas sobre sus cauces. (Ver Plano $\mathrm{N}^{\circ} 2$ ).

En el modelo territorial se muestra como tanto las quebradas como los ríos unen las zonas de conservación activa con fines forestales, educativos-ambientales, agroecológicos, histórico-culturales y la zona de conservación estricta entre sí; así como también éstos se conectan con las áreas verdes urbanas más grandes como los parques de la Madre y del Paraíso. Por lo tanto, esta categoría se plantea con el enfoque de "corredor biológico", cuyas actividades compatibles serán las de recreación activa y pasiva, siguiendo el modelo ya existente en la ciudad aplicado al río Yanuncay. Su recorrido será además la base de caminos peatonales y ciclovías (Ver Plano $\mathrm{N}^{\circ} 2$ ).

H. Zonas de explotación agroecológica periurbana: Representa el $22,1 \%$ con una superficie de 1147,59 ha. Es la aplicación de la agroecología al espacio periurbano medianamente artificializado. En este caso, el territorio se encuentra en proceso de consolidación; sin embargo, su valor agrícola justifica detener la urbanización para aplicar un modelo diferente que permita el óptimo aprovechamiento del recurso suelo.

Para ello es necesario propender a una fragmentación mínima igual al lote óptimo productivo, para garantizar el aprovechamiento del recurso y asegurar la rentabilidad de la agricultura. (Fernández, 1996) Por lo tanto, considerando que es una zona en la cual se busca recuperar y fortalecer tal actividad primaria no se podrá subdividir el suelo en parcelas menores a $2500 \mathrm{~m} 2$, correspondientes al predio óptimo de producción. Para el caso de predios con superficies menores se propone la asociación entre familias vecinas mediante el modelo de "agricultura familiar".

Según la FAO (Food and Agriculture Organization) lo que caracteriza a la "agricultura familiar" es: la utilización de mano de obra familiar, en donde la explotación del predio depende directa y principalmente de la vinculación de la fuerza de trabajo familiar, sin perjuicio del empleo ocasional en otras actividades o de la contratación de mano de obra temporal; el lugar de vivienda coincide con el lugar en donde se desarrolla la actividad productiva; en cuanto a la fuente del ingreso económico, la mayor proporción del ingreso deberá provenir de la explotación agroecológica; y en lo que respecta a la comercialización de la producción, el destino de la mayor parte de la producción es el mercado local. (Fernández,1996)

Por su parte la edificación de viviendas y equipamientos relacionados a la producción agrícola estará sometida a ciertas condiciones. La principal es mantener las construcciones al borde de las vías con el objetivo de liberar el suelo para un mejor desarrollo agrícola. Las normativas españolas, que tratando de evitar núcleos de consolidación en el suelo rural, han condicionado las características de ocupación con normativas como la determinación de un porcentaje de superficie máxima de construcción con relación al área total del lote, o la otra condición de dejar un determinado radio entre una edificación y otra para evitar la consolidación, resultaron ser contraproducentes al objetivo principal del suelo rural -su aprovechamiento agrícolay fortaleció el fenómeno de dispersión en las zonas rurales y la disminución de la productividad. (Serrano. Ruiz, 2003)

Tomando esa experiencia como referencia se plantea que tanto las edificaciones de vivienda como de equipamientos relacionados con la producción se ubiquen al borde de la vía dejando un respectivo retiro frontal y retiros laterales (Ver Plano $\mathrm{N}^{\circ} 2$ ).

I. Zonas de explotación ganadera periurbana sustentable: Representa el 5,1\% con una superficie de 262,31 ha. Se conoce que la producción ganadera es una de las actividades que mayores daños causa al medio ambiente; así, se estima que la producción de carnes para consumo es responsable del $18 \%$ de las emisiones de gases del efecto invernadero, mayor aún que aquellas causadas por el transporte. Puntualmente en el territorio de estudio se identifica que la pérdida de vegetación nativa y la deforestación en general se deben, en gran parte, al desbroce del suelo para extender las zonas de pastoreo.

Por su parte, la "ganadería sustentable" pretende mantener la misma producción sin causar daños al medio ambiente; es un sistema viable económicamente, soportable en cuanto a las exigencias de trabajo y sociales que supone, transmisible en términos de sucesión generacional y reproducible a largo plazo desde un punto de vista medioambiental. Según esa definición, las características y objetivos del ganadero y de su entorno familiar deben ser considerados decisivos en el desarrollo del sistema de producción sostenible. (Serrano. Ruiz, 2003: 168) 
Por lo tanto, "el concepto de sistema familia-explotación constituye, pues, un marco adecuado para el estudio de la sostenibilidad de los sistemas de producción" (Serrano. Ruiz, 2003) Bajo esos principios se propone producir en estos territorios la crianza de animales mayores como el ganado vacuno, y de menores como: cerdos, ovejas, aves de corral, cuyes, conejos, abejas, etc. Su producción deberá satisfacer parte de la demanda local de la ciudad. Como en el caso de la producción agrícola periurbana, su ubicación inmediata a la ciudad reduce los costos y dificultades de transporte de los productos, e incluso cabe la posibilidad de implantar mercados en las propias zonas de producción.

\section{J. Zonas de explotación turística sustentable: Re-} presenta el $1,8 \%$ con una superficie igual a 93,45 ha. Al ser el turismo una actividad que depende de los recursos naturales, los mismos que representan su razón de ser y parte de su atractivo, es indispensable contemplar los principios de sustentabilidad al momento de implantar actividades turísticas sobre el territorio. Es decir; una buena gestión del turismo exige garantizar la sostenibilidad de los recursos de los que depende.

Así, el turismo sustentable se presenta como una estrategia de desarrollo económico local, entre sus ventajas tenemos que: los recursos naturales y culturales se conservan para su uso continuado en el futuro, al tiempo que reportan beneficios; el desarrollo turístico se planifica y gestiona de forma que no causa serios problemas ambientales o socioculturales; la calidad ambiental de la ciudad mejora y se mantiene; los beneficios del turismo se reparten ampliamente entre toda la sociedad. Por lo tanto, se propone aprovechar la calidad paisajística y la geomorfología que posee esta zona, fomentando el turismo relacionado a la práctica de diferentes actividades al aire libre o deportes de aventura, como el montañismo, trekking, acampada, ciclismo de montaña, cabalgatas, caminatas, etc., cuyo valor radica en un espacio físico en su estado natural y con instalaciones construidas muy ligeras y respetuosas con el medio.

\section{K. Zonas de expansión urbana con restricción de} tipo geomorfológico: Representa el $13,1 \%$ con una superficie de 681,82 ha. Esta categoría corresponde a suelos sin vocación definida, por lo cual se asigna como zona de expansión urbana; sin embargo, su geomorfología caracterizada por suelo con pendientes, que si bien son menores del 30\%, no dejan de ser limitantes que condicionan la construcción de edificaciones. Por ese motivo, la urbanización se restringe y se vuelve necesario buscar sistemas constructivos, materiales y morfología alternativos y acordes al medio físico que permitan mantener la armonía visual, gozar de un paisaje de calidad, garantizar el correcto drenaje del agua lluvia, y en definitiva, mejorar la calidad de vida. Es necesario encontrar un tipo de intervención arquitectónica que contemple las dificultades que presenta la construcción en predios con pendiente aplicado al territorio de estudio. Este planteamiento se presenta como un tema de análisis que tarde o temprano deberá ser mirado por los arquitectos y urbanistas en busca de soluciones estéticas, funcionales y sensibles con el medio para lo que será la expansión de la ciudad de Cuenca, pues tanto al Norte, Este y Oeste como en el Sur los suelos presentan las mismas características.

Al norte del predio en el que se implantará la cárcel, paralelo a la vía que lleva a dicho equipamiento, se determina como de expansión urbana con restricciones de tipo geomorfológico, pensando en el impacto que causará el equipamiento y la inevitable urbanización a lo largo de la vía; no obstante, si bien se permite la edificación también es cierto que deberá ser bajo ciertas condiciones para evitar que crezca la amenaza hacia la ZPN Agua Santa; junto a esta categoría se delimita una zona de amortiguamiento (Ver Plano $\mathrm{N}^{\circ} 2$ ).

L. Asentamientos urbanos Tipo A: Representa el 1,5\% con una superficie de 75,62 ha y corresponde a Huizhil y a San José de Baños; en el primer caso el asentamiento se ubica en la ZPN Loma de Huizhil cuya categoría corresponde a la de Conservación Activa con fines ambientales-educativos, para el segundo caso está ubicado en su perímetro inmediato. Sin embargo, ambos asentamientos atentan contra el objetivo de conservación de la ZPN, por lo que se propone detener su expansión territorial y su incremento poblacional.

En el caso de Huizhil, sus pobladores se integrarán a los objetivos de conservación, manteniéndose las actividades agrícolas existentes pero sujetas a los principios de la agroecología que serán socializados al público como parte de la educación ambiental. Se prohíbe la subdivisión del suelo y todo tipo de construcción particular e independiente de los objetivos de conservación de la ZPN. Ventajosamente no habita el $100 \%$ de la población de Huizhil dentro de la ZPN, lo que en última instancia, si no se llega a un consenso, facilitaría la reubicación de los pobladores. En el caso de San José de Baños también se prohíbe la subdivisión del suelo y todo tipo de construcción particular; se propone aplicar la agroecología periurbana y el modelo de producción familiar (Ver Plano $\mathrm{N}^{\circ} 2$ ). 
M. Asentamientos urbanos Tipo B: Representa el 5,2\% con una superficie de 267,97 ha y corresponde a las cabeceras parroquiales de San Joaquín, Baños y Turi, y a los asentamientos del Guzho y Tres Marías. Esta categoría propone consolidar su área urbana para concentrar a la nueva población. Dotar de infraestructura, servicios básicos y equipamientos es de suma importancia para incentivar a los pobladores a ubicarse dentro del perímetro; pero, es necesario controlar el precio del suelo para evitar alzas que en vez de concentrar población podría causar el efecto contrario y dispersarla aún más. Es preciso elaborar un plan parcial para la ordenación de estos territorios y garantizar su crecimiento ordenado, el mejoramiento de la calidad de vida de los habitantes del espacio periurbano y la disminución de las amenazas hacia las zonas de conservación activa (Ver Plano $\mathrm{N}^{\circ} 2$ ).

\subsubsection{Normativa de uso y ocupación del suelo}

La normativa se aplica a las siguientes categorías de ordenación: a) Zona de conservación activa con fines educativos-ambientales correspondiente a la ZPN Loma de Huizhil, b) Zona de conservación activa con fines histórico-culturales correspondiente a las ZPN Cerro Monjas y Agua Santa, c) Zona de regeneración y conservación de las cuencas de inundación del recurso hídrico de ríos y quebradas con fines ecológicos y recreativos, y d) Áreas de amortiguamiento de las zonas de conservación activa. Existen dos tipos de normas, unas generales y otras particulares. Todas ellas se caracterizan por establecer las condiciones que garanticen la sustentabilidad de las actividades sobre cada territorio.

\subsubsection{Programas y Proyectos}

Para la consecución de la imagen objetivo no es suficiente establecer normas de tipo preventivo que garanticen el desarrollo de las actividades humanas de acuerdo a la capacidad de acogida del territorio; sino además se propone un sistema de programas y proyectos que aprovechen correctamente los recursos naturales en pos de una conservación activa; todos ellos se resumen en el Cuadro $\mathrm{N}^{\circ} 1$.

\subsubsection{Modelo de Gestión de las ZPN}

La gestión del territorio es la fase final de un plan de ordenamiento y consiste en la puesta en marcha, el seguimiento y el control de las determinaciones del plan. Fundamentalmente, su propuesta reside en el diseño de un "ente gestor" y su funcionamiento. La estructura del ente gestor se resume en el Gráfico No. 3.

\subsubsection{La "custodia del territorio" como un modelo de gestión de las ZPN}

La custodia del territorio se define como "un conjunto de estrategias y técnicas diversas que pretenden favorecer y hacer posible la responsabilidad de los propietarios o usuarios del territorio en la conservación de sus valores naturales, culturales y paisajísticos y en el uso adecuado (sostenible) de sus recursos" (Donada, 2005 : 7)

Se creará por lo tanto, un documento legal o título de conservación mediante el cual los propietarios puedan hacer uso normal de su propiedad, como venderla producir o vivir en ella, pero comprometiéndose a cumplir con la normativa establecida para las ZPN, con la intención de proteger las áreas de valor y cumplir con los objetivos del Plan de Ordenamiento de las ZPN de la microcuenca del río Tarqui. A cambio, mediante un convenio de colaboración, la municipalidad o ente de custodia llevará a cabo un programa de educación ambiental que implica también la asistencia técnica para mejorar la producción agrícola existente. Adicionalmente la asociación de propietarios, institución con la que se firma el convenio, será acreedora de las denominadas deducciones tributarias, que consisten en la reducción o exoneración de los tributos municipales como las tarifas de servicios básicos, contribución especial de mejoras y rentas patrimoniales, etc. En la normativa se explican en detalle las reducciones o exoneraciones para cada caso posible.

El modelo propuesto es muy ambicioso, su dificultad principal radica en llegar a concretar acuerdos con los propietarios de los predios y hacer un seguimiento para su cumplimiento; sin embargo, todo dependerá de la habilidad de la administración municipal al llevar a cabo este nuevo modelo de gestión, en el cual debe existir un objetivo claro y común, tanto para el ente de custodia (municipalidad) como para el propietario del predio: la conservación de las ZPN en pos de lograr un desarrollo sustentable en la ciudad de Cuenca. La ganancia en dicho convenio deberá ser mutua, y en conjunto, el modelo expuesto implica una mejor calidad de vida tanto para los habitantes de las ZPN como para el resto de la ciudad, de los cuales estos últimos tendrían libre acceso al disfrute que ofrece el territorio.

El modelo se aplica a las tres ZPN, así de manera puntual tenemos que:

- En la ZPN Agua Santa el $42 \%$ del suelo (62,86 ha) es de tenencia municipal y el $58 \%$ restante $(86,24$ ha) se gestionará aplicando este modelo de custodia.

- En el Cerro Monjas, en cambio, el suelo es de tenencia comunal, lo que facilita llegar a un acuerdo para su conservación.

- En la loma de Huizhil existen mayores dificultades por estar 


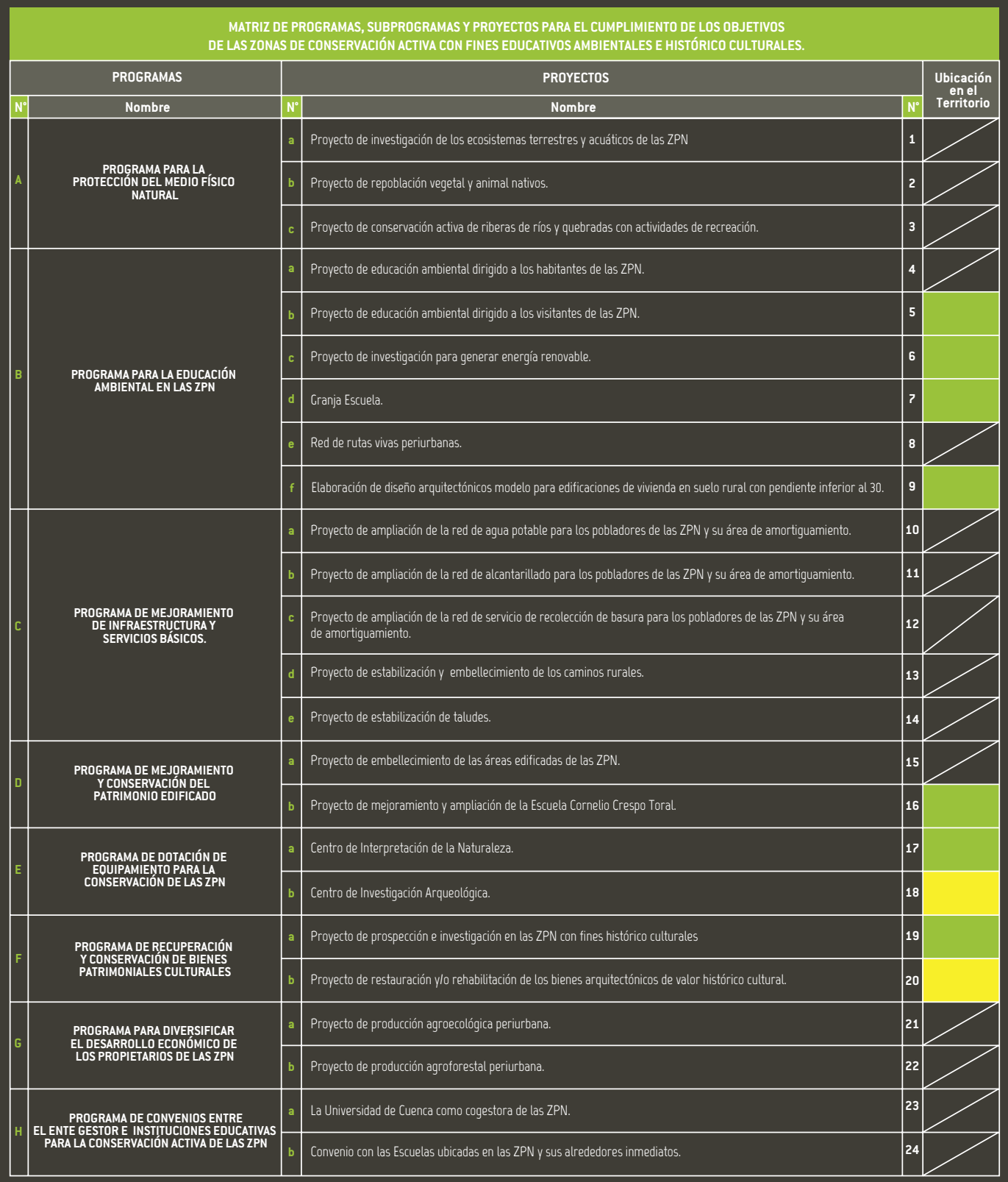

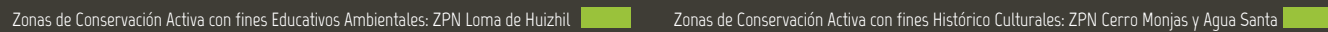
Zonas de Conservación Activa con fines Histórico Culturales: ZPN Loma de Huizhil, Cerro Monjas y Agua Santa

Cuadro $\mathbf{N}^{\circ}$ 1. Matriz de programas y proyectos Fuente y Elaboración: Autora de Tesis

más fragmentada y por la presencia de mayor población en comparación con las otras zonas; sin embargo, se deberá duplicar el esfuerzo en la socialización por parte del ente gestor con los propietarios. La estructura del órgano político del ente gestor que incluye a los presidentes de las juntas parroquiales involucradas y a un representante de la población que habita en cada una de las ZPN permitirá conocer las aspiraciones de sus habitantes y facilitará llegar a un consenso. No se descarta la posibilidad de adquirir el predio, sobre todo en los casos de construcción de equipamiento necesario, y como ya se ha dicho, cabe la posibilidad de que las aspiraciones del propietario no coincidan con los del ente gestor y prefiera vender su predio.

Tanto el título de conservación y el convenio de colaboración se establecerán en base a los objetivos de conservación activa aplicados a cada territorio y en cumplimiento con las normas generales y particulares de los mismos. 

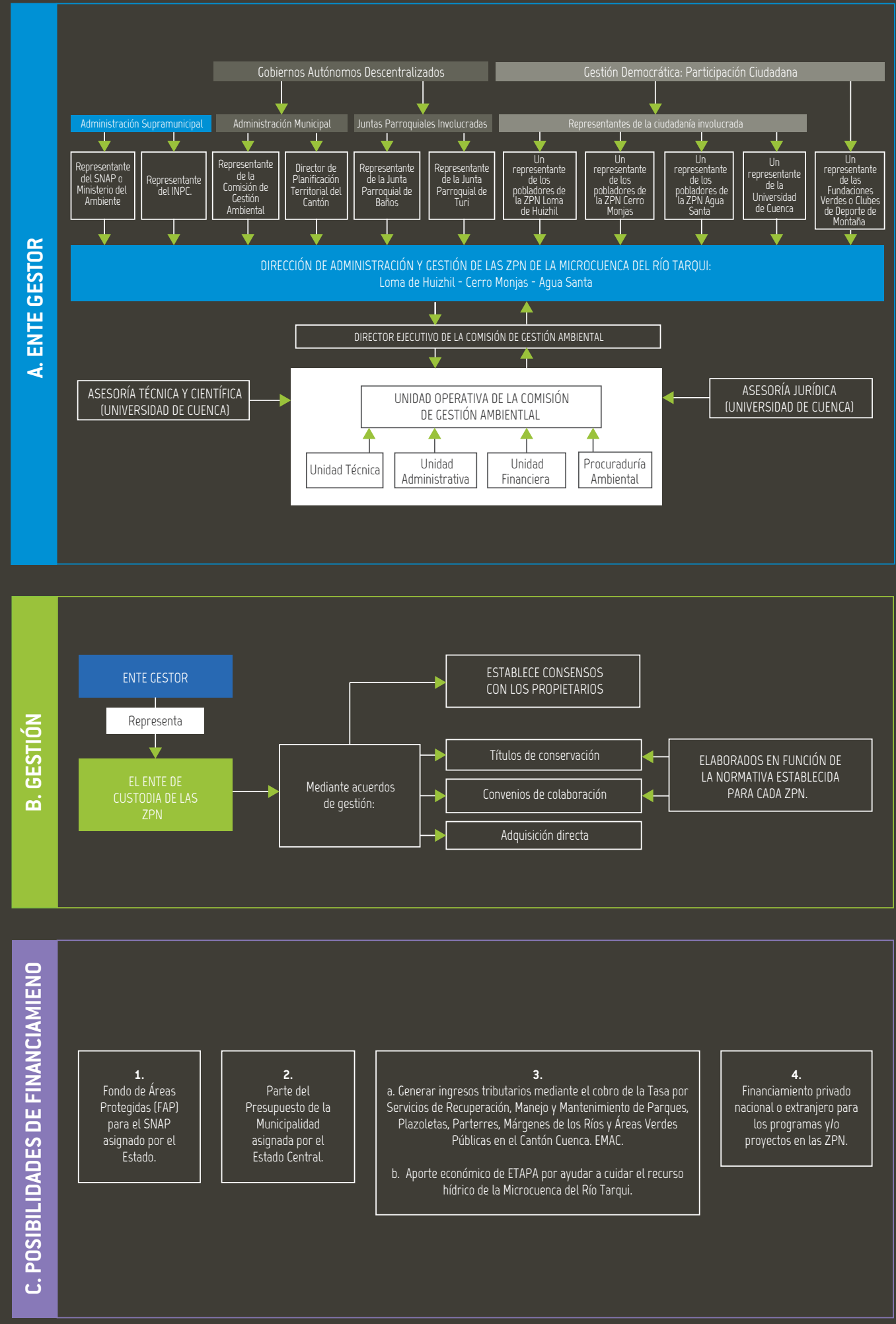

Gráfico $N^{\circ}$ 3. Resumen del modelo de gestión de las ZPN Fuente y elaboración: Autora de Tesis 


\section{Bibliografía:}

Acosta, Luis Alejandro. Rodríguez, Marcos Sebastián (). La Agricultura Familiar en América Latina.

Asamblea Nacional del Ecuador (2008). Constitución del Ecuador. Quito

Asamblea Nacional del Ecuador (2011) Código Orgánico de Organización Territorial, Autonomía y Descentralización COOTAD. Quito: Corporación de Estudios y Publicaciones.

Donada, Laura. Ormazabal, Maya (2005). Custodia del Territorio. Un Modelo de Gestión dentro de Red Natura 2000. Madrid.

Fernández, Gerardo (1996). Estudio sobre el Urbanismo y la Protección de los Recursos Naturales. Madrid: Centro de Publicaciones del Ministerio de Fomento.

Internet (2012). Educación Ambiental. http:// es.wikipedia.org/wiki/Educaci\%С3\%B3n_ambiental. Febrero 2012.

Ministerio del Ambiente (2007). Libro Verde del Medio Ambiente Urbano. Barcelona

Nilson, Kjell. Randrup, Thomas (1997). Silvicultura urbana y periurbana. Antalya. Congreso Forestal Mundial.
Rueda, Salvador (2003). Modelos Urbanos de Ocupación del Territorio: La ciudad compacta y la ciudad difusa. .Barcelona: http://habitat.aq.upm.es/boletin/ n32/asrue.html

Salvador, Pedro J (2003). La Planificación Verde en las Ciudades. Barcelona: Gustavo Gili Ferras, Carlos (2000). Ciudad Dispersa, Aldea Virtual y Revolución Tecnológica. Reflexión acerca de sus relaciones y significado social. Barcelona: Scripta Nova.

Serrano, Emma. Ruiz, Ángel. (2003). Bases para un desarrollo ganadero sostenible: la consideración animal desde una perspectiva sistémica y el estudio de la diversidad de las explotaciones. León.

Unión Internacional para la Conservación de la $\mathrm{Na}$ turaleza UICN (1984). Directrices para las Categorías de Manejo de Áreas Protegidas. Ecuador.

United States Agency International Development (2009). ESTUDIO DE FACTIBILIDAD DE CORREDORES DE CONSERVACIÓN. Ecuador. 